\title{
Inequalities on Generalized Tensor Functions with Diagonalizable and Symmetric Positive Definite Tensors
}

\author{
Maolin Che ${ }^{1}$, Dragana S. Cvetković Ilić ${ }^{2}$, Yimin Wei ${ }^{3, *}$ \\ ${ }^{1}$ School of Economic Mathematics, Southwest University of Finance and Economics, China \\ ${ }^{2}$ Department of Mathematics, Faculty of Science and Mathematics, University of Nis, Serbia \\ ${ }^{3}$ School of Mathematical Sciences and Shanghai Key Laboratory of Contemporary Applied Mathematics, Fudan University, China
}

\begin{abstract}
The main purpose of this paper is to investigate inequalities on symmetric sums of diagonalizable and positive definite tensors. In particular, we generalize the well-known Hlawka and Popoviciu inequalities to the case of diagonalizable and positive definite tensors. As corollaries, we extend Hlawka and Popoviciu inequalities for the combinatorial determinant, permanent and immanant of tensors, and generalized tensor functions.
\end{abstract}

Keywords Diagonalizable tensors, Symmetric positive definite tensors, Generalized tensor functions, Hlawka type inequality; Popoviciu type inequality; Strong superadditivity

AMS 2010 subject classifications 15A39, 15A69, 65A15, 46M05, 47A63

DOI: $10.19139 /$ soic.v6i4.599

\section{Introduction}

Let $\mathbb{G}$ be a subgroup of the symmetric group $\mathbb{S}_{I}$ on the set $\{1,2, \ldots, I\}$ and $\chi$ be an irreducible character of $\mathbb{G}$. The $\mathbb{G}$-immanant (also known as the generalized matrix function [20,22]) of $\mathbf{A} \in \mathbb{R}^{I \times I}$ is defined as

$$
d_{\chi}^{\mathbb{G}}(\mathbf{A})=\sum_{\sigma \in \mathbb{G}} \chi(\sigma) \prod_{i=1}^{I} a_{i \sigma(i)} .
$$

When $\mathbb{G}=\mathbb{S}_{I}, \chi(\sigma)=\operatorname{sgn}(\sigma)$ yields the determinant and $\chi(\sigma) \equiv 1$ yields the permanent.

Macrcus and Minc [21] revealed a relationship between the generalized matrix function and a function involving the eigenvalues of normal matrices and also considered the relationship between the generalized matrix function and an appropriate function of the singular values of an arbitrary square matrix. Berndt and Sra [3] obtained generalized Hlawka and Popoviciu inequalities for generalized matrix functions with positive definite operators. Huang et al. [14] derived inequalities on no-integer power of products of generalized matrix functions on the sum of positive semi-definite matrices. Chang et al. [5] presented an inequality for Kronecker product (sometimes called tensor product) of positive operators on Hilbert spaces and then applied the inequality to generalized matrix functions. Paksoy et al. [24] obtained some inequalities for generalized matrix functions of positive semi-definite matrices by an embedding and through kronecker products.

*Correspondence to: Yimin Wei (Email: ymwei@fudan.edu.cn and yimin.wei@gmail.com.). School of Mathematical Sciences and Shanghai Key Laboratory of Contemporary Applied Mathematics, Fudan University, Shanghai, P. R. of China (200433).

ISSN 2310-5070 (online) ISSN 2311-004X (print)

Copyright (C) 2018 International Academic Press 
Higher-order equivalents of vectors (first order) and matrices (second order) are called higher-order tensors, multi-dimensional matrices, or multi-way arrays. A tensor is an $N$-dimensional array of real numbers denoted by script notation $\mathcal{A} \in \mathbb{R}^{I_{1} \times I_{2} \times \cdots \times I_{N}}$ with entries given by

$$
a_{i_{1} i_{2} \ldots i_{N}} \in \mathbb{R} \quad \text { for } \quad i_{n}=1,2, \ldots, I_{n} \text { and } n=1,2, \ldots, N .
$$

Che et al. [6] defined the generalized tensor function, which is generalized from generalized matrix functions, and indicated that the combinatorial determinant and the permanent of tensors are two special cases of generalized tensor functions. The interested readers can refer to [4, 18, 23, 27] for the combinatorial determinant of tensors and [1, 2, 10, 28, 29] for the permanent of tensors. In this paper, we consider the inequalities on generalized tensor functions with diagonalizable and symmetric positive definite tensors, which can be viewed as the generalization from the inequalities on generalized matrix functions with positive definite matrices.

The following notations will be used throughout this paper. We assume that $I, J$, and $N$ will be reserved to denote the index of upper bounds, unless stated otherwise. We use small letters $x, u, v, \ldots$ for scalars, small bold letters $\mathbf{x}, \mathbf{u}, \mathbf{v}, \ldots$ for vectors, bold capital letters $\mathbf{A}, \mathbf{B}, \mathbf{C}, \ldots$ for matrices, and calligraphic letters $\mathcal{A}, \mathcal{B}, \mathcal{C}, \ldots$ for higher-order tensors. These notations are consistently used for lower-order parts of a given structure. For example, the entry with row index $i$ and column index $j$ in a matrix $\mathbf{A}$, i.e., $(\mathbf{A})_{i j}$, is symbolized by $a_{i j}$ (also $(\mathbf{x})_{i}=x_{i}$ and $(\mathcal{A})_{i_{1} i_{2} \ldots i_{N}}=a_{i_{1} i_{2} \ldots i_{N}}$ ). For some indices, we use MATLAB notation, e.g., the form $i=1: 2: 2 I-1$ meaning that $i$ increases in steps of 2 , taking on only the values $1,3, \ldots, 2 I-1$.

The rest of our paper is organized as follows. In Section 2, we introduce some basic definitions and operators about tensors, such as diagonal tensors, symmetric tensors, diagonalizable tensors, Kronecker product of tensors, and so on. In Section 3, we introduce the definition of generalized tensor functions and indicate that the permanent and the combinatorial determinant of tensors are two special cases. In this section, we also consider the basic properties on the Kronecker product of tensors. Three different kinds of inequalities on generalized tensor functions associated with the diagonalizable and symmetric positive definite tensors are considered in Sections 4, 5 and 6, respectively.

\section{Preliminaries}

If we set $I_{n}:=I$, then the set of order $N$ dimension $I$ tensors will be denoted by $T_{N, I}$. The mode- $n$ product [15] of a complex tensor $\mathcal{A} \in T_{N, I}$ by a matrix $\mathbf{B} \in \mathbb{R}^{I \times I}$, denoted by $\mathcal{A} \times{ }_{n} \mathbf{B}$, is a tensor $\mathcal{C} \in T_{N, I}$, and its entries will be given by

$$
c_{i_{1} \ldots i_{n-1} j i_{n+1} \ldots i_{N}}=\sum_{i_{n}=1}^{I} a_{i_{1} i_{2} \ldots i_{N}} b_{j i_{n}},
$$

where $n=1,2, \ldots, N$.

In particular, the mode- $n$ multiplication of a real tensor $\mathcal{A} \in T_{N, I}$ by a vector $\mathbf{z} \in \mathbb{R}^{I}$ is denoted by $\mathcal{A} \times{ }_{n} \mathbf{z}^{\top}$. If we set $\mathcal{C}=\mathcal{A} \times{ }_{n} \mathbf{z}^{\top} \in T_{N-1, I}$, then we have element-wise [15],

$$
c_{i_{1} \ldots i_{n-1} i_{n+1} \ldots i_{N}}=\sum_{i_{n}=1}^{I} a_{i_{1} \ldots i_{n-1} i_{n} i_{n+1} \ldots i_{N}} x_{i_{n}} .
$$

For any given tensor $\mathcal{A} \in T_{N, I}$ and the matrices $\mathbf{F}, \mathbf{G} \in \mathbb{R}^{I \times I}$, one has [15]

$$
\left(\mathcal{A} \times{ }_{m} \mathbf{F}\right) \times_{n} \mathbf{G}=\left(\mathcal{A} \times{ }_{n} \mathbf{G}\right) \times_{m} \mathbf{F}=\mathcal{A} \times{ }_{m} \mathbf{F} \times{ }_{n} \mathbf{G}, \quad\left(\mathcal{A} \times{ }_{n} \mathbf{F}\right) \times_{n} \mathbf{G}=\mathcal{A} \times{ }_{n}(\mathbf{G} \cdot \mathbf{F}),
$$

where '.' means the multiplication of two matrices with different integers $m$ and $n$. 
We introduce the following two notations from [25]. For any $\mathcal{A} \in T_{N, I}$ and $\mathrm{x} \in \mathbb{R}^{I}, \mathcal{A} \mathrm{x}^{N-1}$ is an $I$ dimensional real vector whose $i$ th component is

$$
\left(\mathcal{A} \mathbf{x}^{N-1}\right)_{i}=\sum_{i_{2}, \ldots, i_{N}=1}^{I} a_{i i_{2} \ldots i_{N}} x_{i_{2}} \ldots x_{i_{N}}
$$

and $\mathcal{A} \mathrm{x}^{N}$ is a scalar given by

$$
\mathcal{A} \mathbf{x}^{N}=\sum_{i_{1}, i_{2}, \ldots, i_{N}=1}^{I} a_{i_{1} i_{2} \ldots i_{N}} x_{i_{1}} x_{i_{2}} \ldots x_{i_{N}} .
$$

A tensor $\mathcal{A} \in T_{N, I}$ is symmetric, if $a_{i_{1} i_{2} \ldots i_{N}}$ is invariant by any permutation $\pi \in \mathbb{S}_{I}$, that is, $a_{i_{1} i_{2} \ldots i_{N}}=$ $a_{i_{\pi(1)} i_{\pi(2)} \ldots i_{\pi(N)}}$ where $i_{n}=1,2, \ldots, I$ and $n=1,2, \ldots, N$. The set of all symmetric tensors in $T_{N, I}$ is denoted by $S T_{N, I}$. A tensor $\mathcal{D} \in T_{N, I}$ is diagonal [15] if $d_{i_{1} i_{2} \ldots i_{N}}=0$, where $i_{1}, \ldots, i_{N}$ are not identical for $i_{n}=$ $1,2, \ldots, I$ and $n=1,2, \ldots, N$. In particular, for a diagonal tensor $\mathcal{D} \in T_{N, I}$, if $d_{i i \ldots i}=1$ for $i=1,2, \ldots, I$, then $\mathcal{D}$ is called the identity tensor, and it is denoted by $\mathcal{I}$. We now introduce the definition of diagonalizable symmetric tensors in $T_{N, I}$.

Definition 2.1

([7, Definition 2.5]) Suppose that $\mathcal{A} \in T_{N, I}$ is symmetric. $\mathcal{A}$ is called diagonalizable symmetric if and only if $\mathcal{A}$ can be represented as

$$
\mathcal{A}=\mathcal{D} \times{ }_{1} \mathbf{B} \times{ }_{2} \mathbf{B} \cdots \times{ }_{N} \mathbf{B},
$$

where $\mathbf{B} \in \mathbb{R}^{I \times I}$ and $\mathcal{D} \in T_{N, I}$ is diagonal. The set of all diagonalizable symmetric tensors in $S T_{N, I}$ is denoted by $D_{N, I}$.

More general, we have the following definition.

Definition 2.2

Suppose that $\mathcal{A} \in T_{N, I}$. $\mathcal{A}$ is called diagonalizable if $\mathcal{A}$ can be represented as

$$
\mathcal{A}=\mathcal{D} \times{ }_{1} \mathbf{B}_{1} \times{ }_{2} \mathbf{B}_{2} \cdots \times{ }_{N} \mathbf{B}_{N},
$$

where $\mathbf{B}_{n} \in \mathbb{R}^{I \times I}$ with $n=1,2, \ldots, N$, and $\mathcal{D} \in T_{N, I}$ is diagonal.

In this paper, we suppose that $N$ is even. For a given $\mathcal{A} \in S T_{N, I}$, if $\mathcal{A} \mathrm{x}^{N}>0$ for all nonzero $\mathrm{x} \in \mathbb{R}^{I}$, then $\mathcal{A}$ is positive definite; if $\mathcal{A} \mathrm{x}^{N} \geq 0$ for all $\mathrm{x} \in \mathbb{R}^{I}$, then $\mathcal{A}$ is positive semi-definite. Suppose that $\mathcal{A}=$ $\mathcal{D} \times{ }_{1} \mathbf{B} \times{ }_{2} \mathbf{B} \cdots \times{ }_{N} \mathbf{B}$, we indicate the following statements:

a) if $\mathcal{D}$ is positive semi-definite, then so is $\mathcal{A}$;

b) if $\mathcal{D}$ is positive semi-definite with all diagonal entries nonzero, and $\mathbf{B}$ is nonsingular, then $\mathcal{A}$ is positive definite and is called a completely decomposable tensor [17];

c) if $\mathcal{D}$ is positive semi-definite with all diagonal entries nonnegative, and $\mathbf{B}$ is nonnegative, then $\mathcal{A}$ is called a completely positive tensor. For the properties and checkability of completely positive tensors, the interested readers are referred to $[11,19,26,30,31]$ and the references therein.

If $\mathcal{A} \mathrm{x}^{N}$ can be decomposed to the sum of squares of polynomial of degree $N / 2$, then $\mathcal{A} \mathrm{x}^{N}$ is call a sumof-squares (SOS) polynomial, and the corresponding symmetric tensor $\mathcal{A}$ is called an SOS tensor [13]. Some important properties of SOS tensors can be referred to $[8,9,17]$ and the references therein. Note that the set of all SOS tensors equal to the set of all completely decomposable tensors with an even $N$.

For two given $\mathcal{A}, \mathcal{B} \in S T_{N, I}$, the operator inequality $\mathcal{A} \geq \mathcal{B}$ denotes the Löwner partial order [3], meaning that $\mathcal{A}-\mathcal{B}$ is positive definite. 


\section{Kronecker product of tensors and Generalized tensor functions}

The Kronecker product [16] of $\mathcal{A}, \mathcal{B} \in T_{N, I}$ yields a tensor $\mathcal{C}=\mathcal{A} \otimes \mathcal{B}: \equiv \mathcal{A B} \in T_{N, I^{2}}$ with entries

$$
\mathcal{C}\left(j_{1}+\left(i_{1}-1\right) I, j_{2}+\left(i_{2}-1\right) I, \ldots, j_{N}+\left(i_{N}-1\right) I\right)=a_{i_{1} i_{2} \ldots i_{N}} b_{j_{1} j_{2} \ldots j_{N}}
$$

where $i_{n}, j_{n}=1,2, \ldots, I$ with $n=1,2, \ldots, N$. Wherever multiplication is used, we mean Kronecker products (though unusual, we use this notation for esthetic reasons to keep the "visual burden" of our proofs low); thus for arbitrary tensors $\mathcal{A}$ and $\mathcal{B}$ :

$$
\begin{aligned}
\mathcal{A}^{P} & \equiv \otimes^{P} \mathcal{A}=\mathcal{A} \otimes \mathcal{A} \otimes \cdots \otimes \mathcal{A}, \\
\mathcal{A}^{P} \mathcal{B}^{Q} & \equiv\left(\otimes^{P} \mathcal{A}\right) \otimes\left(\otimes^{Q} \mathcal{B}\right),
\end{aligned}
$$

where $P \geq 1$ and $Q \geq 1$ are two given positive integers. Note that this multiplication is nocommutative, so $\mathcal{A B} \neq \mathcal{B} \mathcal{A}$.

Since the entire paper relies extensively on elementary properties of Kronecker products, let us briefly recall these below.

Proposition 3.1

Let $\mathcal{A}, \mathcal{B}, \mathcal{E}, \mathcal{F} \in D_{N, I}$ be positive definite with an even $N$. Then we have the following results.

(i) $\mathcal{A B} \equiv \mathcal{A} \otimes \mathcal{B}$ is also positive definite;

(ii) If $\mathcal{A} \geq \mathcal{B}$ and $\mathcal{E} \geq \mathcal{F}$, then $\mathcal{A E} \geq \mathcal{B F}$;

(iii) $\mathcal{A}(\mathcal{B}+\mathcal{E})=\mathcal{A B}+\mathcal{A} \mathcal{E}$ and $(\mathcal{A}+\mathcal{B}) \mathcal{E}=\mathcal{A E}+\mathcal{B E}$;

(iv) $(\mathcal{A}+\mathcal{B})^{P} \geq \mathcal{A}^{P}+\mathcal{B}^{P}$ where $P \geq 1$ is any positive integer.

Proof

According to the assumption, we can rewrite $\mathcal{A}, \mathcal{B}, \mathcal{E}, \mathcal{F}$ as

$$
\begin{aligned}
& \mathcal{A}=\mathcal{D}_{1} \times{ }_{1} \mathbf{B}_{1} \times{ }_{2} \mathbf{B}_{1} \cdots \times{ }_{N} \mathbf{B}_{1}, \quad \mathcal{B}=\mathcal{D}_{2} \times{ }_{1} \mathbf{B}_{2} \times{ }_{2} \mathbf{B}_{2} \cdots \times{ }_{N} \mathbf{B}_{2}, \\
& \mathcal{E}=\mathcal{D}_{3} \times{ }_{1} \mathbf{B}_{3} \times{ }_{2} \mathbf{B}_{3} \cdots \times{ }_{N} \mathbf{B}_{3}, \quad \mathcal{F}=\mathcal{D}_{4} \times{ }_{1} \mathbf{B}_{4} \times{ }_{2} \mathbf{B}_{4} \cdots \times{ }_{N} \mathbf{B}_{4},
\end{aligned}
$$

where $\mathcal{D}_{i} \in D_{N, I}$ are positive definite and $\mathbf{B}_{i} \in \mathbb{R}^{I \times I}$ are nonsingular for $i=1,2,3,4$.

[Proof of (i)] According to the Kronecker product of tensors, we have

$$
\mathcal{A B} \equiv \mathcal{A} \otimes \mathcal{B}=\left(\mathcal{D}_{1} \otimes \mathcal{D}_{2}\right) \times_{1}\left(\mathbf{B}_{1} \otimes \mathbf{B}_{2}\right) \times_{2}\left(\mathbf{B}_{1} \otimes \mathbf{B}_{2}\right) \cdots \times \times_{N}\left(\mathbf{B}_{1} \otimes \mathbf{B}_{2}\right) .
$$

For any nonzero $\mathrm{x} \in \mathbb{R}^{I^{2}}$, we have

$$
(\mathcal{A B}) \mathbf{x}^{N}=\left(\mathcal{D}_{1} \otimes \mathcal{D}_{2}\right) \times_{1}\left(\mathbf{x}^{\top}\left(\mathbf{B}_{1} \otimes \mathbf{B}_{2}\right)\right) \times_{2}\left(\mathbf{x}^{\top}\left(\mathbf{B}_{1} \otimes \mathbf{B}_{2}\right)\right) \cdots \times_{N}\left(\mathbf{x}^{\top}\left(\mathbf{B}_{1} \otimes \mathbf{B}_{2}\right)\right)>0 .
$$

Hence $\mathcal{A B}$ is positive definite.

[Proof of (ii)] Since $\mathcal{A} \geq \mathcal{B}$ and $\mathcal{E} \geq \mathcal{F}$, then both $\mathcal{A}-\mathcal{B}$ and $\mathcal{E}-\mathcal{F}$ are positive definite. Since

$$
\mathcal{A E}-\mathcal{B F}=(\mathcal{A}-\mathcal{B}) \mathcal{E}+\mathcal{B}(\mathcal{E}-\mathcal{F})
$$

then by term (i), $\mathcal{A E}-\mathcal{B F}$ is positive definite, that is, $\mathcal{A E} \geq \mathcal{B F}$.

[Proof of (iii)] It is trivial.

[Proof of (iv)] Case $P=1$ is trivial. Suppose that $(\mathcal{A}+\mathcal{B})^{P} \geq \mathcal{A}^{P}+\mathcal{B}^{P}$ holds. Now we consider the case of $P+1$. Since

$$
\begin{aligned}
(\mathcal{A}+\mathcal{B})^{P+1} & =(\mathcal{A}+\mathcal{B})^{P}(\mathcal{A}+\mathcal{B})=(\mathcal{A}+\mathcal{B})^{P} \mathcal{A}+(\mathcal{A}+\mathcal{B})^{P} \mathcal{B} \\
& \geq \mathcal{A}^{P} \mathcal{A}+\mathcal{B}^{P} \mathcal{B}=\mathcal{A}^{P+1}+\mathcal{B}^{P+1}
\end{aligned}
$$

The second equality holds for term (iii) and the inequality holds for $\mathcal{A}+\mathcal{B} \geq \mathcal{A}, \mathcal{A}+\mathcal{B} \geq \mathcal{B}$ and term (i). 
Let $\mathbb{G}$ be a subgroup of the symmetric group $\mathbb{S}_{I}$ on the set $\{1,2, \ldots, I\}$ and $\chi_{n}(n=2,3, \ldots, N)$ be an irreducible character of $\mathbb{G}$. The $\mathbb{G}$-immanant [6] (also known as the generalized tensor function) of a tensor $\mathcal{A} \in T_{N, I}$ is defined by

$$
d_{\chi_{2}, \ldots, \chi_{N}}^{\mathbb{G}}(\mathcal{A})=\sum_{\pi_{2}, \ldots, \pi_{N} \in \mathbb{G}} \chi_{2}\left(\pi_{2}\right) \ldots \chi_{N}\left(\pi_{N}\right) \prod_{i=1}^{I} a_{i \pi_{2}(i) \ldots \pi_{N}(i)} .
$$

According to the definition of generalized tensor functions, it is known that there is a vector $\mathbf{x} \in \mathbb{R}^{I^{I}}$ such that

$$
d_{\chi_{2}, \ldots, \chi_{N}}^{\mathbb{G}}(\mathcal{A})=\left(\mathcal{A}^{I}\right) \mathbf{x}^{N} .
$$

There exist three special cases of generalized tensor functions. One is the combinatorial determinant of the tensor $\mathcal{A} \in T_{N, I}$, denoted by $\operatorname{det}_{c}(\mathcal{A})$, which is defined as

$$
\operatorname{det}_{c}(\mathcal{A})=\sum_{\pi_{2}, \ldots, \pi_{N} \in \mathbb{S}_{I}} \operatorname{sgn}\left(\pi_{N-P+1}\right) \ldots \operatorname{sgn}\left(\pi_{N}\right) \prod_{i=1}^{I} a_{i \pi_{2}(i) \ldots \pi_{N}(i)},
$$

where $\operatorname{sgn}(\pi)$ is the sign of $\pi \in \mathbb{S}_{I}$ and a positive integer $P$ satisfies

$$
P=(N+1) / 2, \text { for an odd } N ; \text { or } P=N / 2 \text {, for an even } N .
$$

Another one is the permanent of the tensor $\mathcal{A} \in T_{N, I}$, denoted by perm $(\mathcal{A})$, defined as

$$
\operatorname{perm}(\mathcal{A})=\sum_{\pi_{2}, \ldots, \pi_{N} \in \mathbb{S}_{I}} \prod_{i=1}^{I} a_{i \pi_{2}(i) \ldots \pi_{N}(i)} .
$$

Finally, let $\lambda=\left(\lambda_{1}, \lambda_{2}, \ldots\right)$ be a partition of $I$ and $\chi_{\lambda}$ be the corresponding irreducible representation theoretic character of the symmetric group $\mathbb{S}_{I}$, the immanant of $\mathcal{A} \in T_{N, I}$ associated with the character $\chi_{\lambda}$ is defined as

$$
\operatorname{Imm}_{\lambda}(\mathcal{A})=\sum_{\pi_{2}, \ldots, \pi_{N} \in \mathbb{S}_{I}} \chi_{\lambda}\left(\pi_{2}\right) \ldots \chi_{\lambda}\left(\pi_{N}\right) \prod_{i=1}^{I} a_{i \pi_{2}(i) \ldots \pi_{N}(i)} .
$$

\section{Hlawka type inequalities and its generalization}

Let $f$ be a convex function on a real interval $\mathbb{I} \subset \mathbb{R}$, if $a, b, c \in \mathbb{I}$, then $f(a+b+c)+f(a)+f(b)+f(c) \geq$ $f(a+b)+f(a+c)+f(b+c)$. This inequality is called the functional Hlawka inequality [12]. In this section, we generalize Hlawka inequality to the case of generalized tensor functions.

With Proposition 3.1, we are ready to prove our first positive definite tensor Hlawka type inequality. In the matrix case, the following theorem is proved in [3, Theorem 2.1].

\section{Theorem 4.1}

Let $\mathcal{A}, \mathcal{B}, \mathcal{C} \in D_{N, I}$ be positive definite with an even $N$. Then for each integer $P \geq 1$, we have

$$
(\mathcal{A}+\mathcal{B}+\mathcal{C})^{P}+\mathcal{A}^{P}+\mathcal{B}^{P}+\mathcal{C}^{P} \geq(\mathcal{A}+\mathcal{B})^{P}+(\mathcal{A}+\mathcal{C})^{P}+(\mathcal{B}+\mathcal{C})^{P} .
$$

Proof

The case of $P=1$ is trivial and holds with equality. Unsurprisingly, for $P=2$, we again have equality, since both sides expend to

$$
2\left(\mathcal{A}^{2}+\mathcal{B}^{2}+\mathcal{C}^{2}\right)+\mathcal{A B}+\mathcal{B A}+\mathcal{A C}+\mathcal{C} \mathcal{A}+\mathcal{B C}+\mathcal{C B} .
$$


We prove the general claim by induction. Assume therefore that (4.1) holds for some integers $P \geq 2$. Then,

$$
\begin{aligned}
(\mathcal{A}+\mathcal{B}+\mathcal{C})^{P+1}= & (\mathcal{A}+\mathcal{B}+\mathcal{C})^{P}(\mathcal{A}+\mathcal{B}+\mathcal{C}) \\
\geq & \left((\mathcal{A}+\mathcal{B})^{P}+(\mathcal{A}+\mathcal{C})^{P}+(\mathcal{B}+\mathcal{C})^{P}-\mathcal{A}^{P}-\mathcal{B}^{P}-\mathcal{C}^{P}\right)(\mathcal{A}+\mathcal{B}+\mathcal{C}) \\
= & (\mathcal{A}+\mathcal{B})^{P+1}+(\mathcal{A}+\mathcal{C})^{P+1}+(\mathcal{B}+\mathcal{C})^{P+1} \\
& -\mathcal{A}^{P+1}-\mathcal{B}^{P+1}-\mathcal{C}^{P+1}+\mathcal{T}
\end{aligned}
$$

where the inequality follows from the induction hypothesis. The term $\mathcal{T}$ is defined as

$$
\mathcal{T}=(\mathcal{A}+\mathcal{B})^{P} \mathcal{C}+(\mathcal{A}+\mathcal{C})^{P} \mathcal{B}+(\mathcal{B}+\mathcal{C})^{P} \mathcal{A}-\mathcal{A}^{P}(\mathcal{B}+\mathcal{C})-\mathcal{B}^{P}(\mathcal{A}+\mathcal{C})-\mathcal{C}^{P}(\mathcal{A}+\mathcal{B})
$$

It remains to show that $\mathcal{T}$ is positive semi-definite. But this follows immediately upon applying the superadditivity inequality to the first three terms of $\mathcal{T}$ and cancelling. Thus, inequality (4.1) is proved.

\section{Corollary 4.1}

Let $\mathcal{A}, \mathcal{B}, \mathcal{C} \in D_{N, I}$ be positive definite with an even $N$. Then for each integer $P \geq 1$,

$$
(\mathcal{A}+\mathcal{B}+\mathcal{C})^{P}+\mathcal{A}^{P} \geq(\mathcal{A}+\mathcal{B})^{P}+(\mathcal{A}+\mathcal{C})^{P} .
$$

Proof

It follows by Proposition 3.1 and inequality (4.1).

\section{Remark 4.1}

The inequality (4.1) is called strong superadditivity of tensor products; readers familiar with combinatorics may recognize it as supermodularity.

In the special case when $N=2$, the following result has been established in [3, Corollary 2.3].

\section{Theorem 4.2}

Let $\mathcal{A}, \mathcal{B}, \mathcal{C} \in D_{N, I}$ be positive definite with an even $N$. Suppose that $d_{\chi_{2}, \ldots, \chi_{N}}^{\mathbb{G}}$ is the generalized tensor function defined on the set $T_{N, I}$. Then

$$
\begin{gathered}
d_{\chi_{2}, \ldots, \chi_{N}}^{\mathbb{G}}(\mathcal{A}+\mathcal{B}+\mathcal{C})+d_{\chi_{2}, \ldots, \chi_{N}}^{\mathbb{G}}(\mathcal{A})+d_{\chi_{2}, \ldots, \chi_{N}}^{\mathbb{G}}(\mathcal{B})+d_{\chi_{2}, \ldots, \chi_{N}}^{\mathbb{G}}(\mathcal{C}) \\
\geq d_{\chi_{2}, \ldots, \chi_{N}}^{\mathbb{G}}(\mathcal{A}+\mathcal{B})+d_{\chi_{2}, \ldots, \chi_{N}}^{\mathbb{G}}(\mathcal{A}+\mathcal{C})+d_{\chi_{2}, \ldots, \chi_{N}}^{\mathbb{G}}(\mathcal{B}+\mathcal{C}) .
\end{gathered}
$$

Proof

Congruence preserves Löwner partial order, so we use (3.2) and (4.1) to derive this theorem.

Suppose that $\mathcal{A}, \mathcal{B}, \mathcal{C} \in D_{N, I}$ are positive definite with an even $N$. Based on Theorem 4.1, for any positive integers $L$ and $P$, we have

$$
\left((\mathcal{A}+\mathcal{B}+\mathcal{C})^{P}\right)^{L}+\left(\mathcal{A}^{P}\right)^{L}+\left(\mathcal{B}^{P}\right)^{L}+\left(\mathcal{C}^{P}\right)^{L} \geq\left((\mathcal{A}+\mathcal{B})^{P}\right)^{L}+\left((\mathcal{A}+\mathcal{C})^{P}\right)^{L}+\left((\mathcal{B}+\mathcal{C})^{P}\right)^{L}
$$

which implies that

$$
\begin{aligned}
& d_{\chi_{2}, \ldots, \chi_{N}}^{\mathbb{G}}(\mathcal{A}+\mathcal{B}+\mathcal{C})^{L}+d_{\chi_{2}, \ldots, \chi_{N}}^{\mathbb{G}}(\mathcal{A})^{L}+d_{\chi_{2}, \ldots, \chi_{N}}^{\mathbb{G}}(\mathcal{B})^{L}+d_{\chi_{2}, \ldots, \chi_{N}}^{\mathbb{G}}(\mathcal{C})^{L} \\
& \geq d_{\chi_{2}, \ldots, \chi_{N}}^{\mathbb{G}}(\mathcal{A}+\mathcal{B})^{L}+d_{\chi_{2}, \ldots, \chi_{N}}^{\mathbb{G}}(\mathcal{A}+\mathcal{C})^{L}+d_{\chi_{2}, \ldots, \chi_{N}}^{\mathbb{G}}(\mathcal{B}+\mathcal{C})^{L} .
\end{aligned}
$$

In general, we have the following conjecture, which shows inequality involving the non-integer powers of generalized tensor functions. 
Conjecture 4.1

Let $\mathcal{A}, \mathcal{B}, \mathcal{C} \in D_{N, I}$ be positive definite with an even $N$. Suppose that $d_{\chi_{2}, \ldots, \chi_{N}}^{\mathbb{G}}$ is the generalized tensor function defined on the set $T_{N, I}$. Then for any $r \in\{1\} \cup[2, \infty)$,

$$
\begin{aligned}
& d_{\chi_{2}, \ldots, \chi_{N}}^{\mathbb{G}}(\mathcal{A}+\mathcal{B}+\mathcal{C})^{r}+d_{\chi_{2}, \ldots, \chi_{N}}^{\mathbb{G}}(\mathcal{A})^{r}+d_{\chi_{2}, \ldots, \chi_{N}}^{\mathbb{G}}(\mathcal{B})^{r}+d_{\chi_{2}, \ldots, \chi_{N}}^{\mathbb{G}}(\mathcal{C})^{r} \\
& \geq d_{\chi_{2}, \ldots, \chi_{N}}^{\mathbb{G}}(\mathcal{A}+\mathcal{B})^{r}+d_{\chi_{2}, \ldots, \chi_{N}}^{\mathbb{G}}(\mathcal{A}+\mathcal{C})^{r}+d_{\chi_{2}, \ldots, \chi_{N}}^{\mathbb{G}}(\mathcal{B}+\mathcal{C})^{r} .
\end{aligned}
$$

\section{Remark 4.2}

When $N=2$, this conjecture is proved by Huang et al. [14, Theorem 2.1].

More general, we have the following theorem, which can be viewed as a generalization of Theorem 4.1.

\section{Theorem 4.3}

Let $\mathcal{A}_{i}, \mathcal{B}_{i}, \mathcal{C}_{i} \in D_{N, I_{i}}$ be positive definite with an even $N$ and $i=1,2, \ldots, M$. Then

$$
\begin{aligned}
& \otimes_{i=1}^{M}\left(\mathcal{A}_{i}+\mathcal{B}_{i}+\mathcal{C}_{i}\right)+\otimes_{i=1}^{M} \mathcal{A}_{i}+\otimes_{i=1}^{M} \mathcal{B}_{i}+\otimes_{i=1}^{M} \mathcal{C}_{i} \\
& \geq \otimes_{i=1}^{M}\left(\mathcal{A}_{i}+\mathcal{B}_{i}\right)+\otimes_{i=1}^{M}\left(\mathcal{A}_{i}+\mathcal{C}_{i}\right)+\otimes_{i=1}^{M}\left(\mathcal{B}_{i}+\mathcal{C}_{i}\right) .
\end{aligned}
$$

Consequently, we have

$$
\begin{aligned}
& \otimes_{i=1}^{M}\left(\left(\mathcal{A}_{i}+\mathcal{B}_{i}+\mathcal{C}_{i}\right)^{I_{i}}\right)+\otimes_{i=1}^{M}\left(\mathcal{A}_{i}^{I_{i}}\right)+\otimes_{i=1}^{M}\left(\mathcal{B}_{i}^{I_{i}}\right)+\otimes_{i=1}^{M}\left(\mathcal{C}_{i}^{I_{i}}\right) \\
& \geq \otimes_{i=1}^{M}\left(\left(\mathcal{A}_{i}+\mathcal{B}_{i}\right)^{I_{i}}\right)+\otimes_{i=1}^{M}\left(\left(\mathcal{A}_{i}+\mathcal{C}_{i}\right)^{I_{i}}\right)+\otimes_{i=1}^{M}\left(\left(\mathcal{B}_{i}+\mathcal{C}_{i}\right)^{I_{i}}\right) .
\end{aligned}
$$

Proof

The proof is similar to one of Theorem 4.1.

\section{Theorem 4.4}

Let $\mathcal{A}_{i}, \mathcal{B}_{i}, \mathcal{C}_{i} \in D_{N, I_{i}}$ be positive definite with an even $N$ and $i=1,2, \ldots, M$. Suppose that $d_{\chi 2}^{\mathbb{G}, i}, \ldots, \chi_{N}$ be the generalized tensor function defined on the set $T_{N, I_{i}}$. Then we have

$$
\begin{gathered}
\prod_{i=1}^{M} d_{\chi_{2}, \ldots, \chi_{N}}^{\mathbb{G}, i}\left(\mathcal{A}_{i}+\mathcal{B}_{i}+\mathcal{C}_{i}\right)+\prod_{i=1}^{M} d_{\chi_{2}, \ldots, \chi_{N}}^{\mathbb{G}, i}\left(\mathcal{A}_{i}\right)+\prod_{i=1}^{M} d_{\chi_{2}, \ldots, \chi_{N}}^{\mathbb{G}, i}\left(\mathcal{B}_{i}\right)+\prod_{i=1}^{M} d_{\chi_{2}, \ldots, \chi_{N}}^{\mathbb{G}, i}\left(\mathcal{C}_{i}\right) \\
\geq \prod_{i=1}^{M} d_{\chi_{2}, \ldots, \chi_{N}}^{\mathbb{G}, i}\left(\mathcal{A}_{i}+\mathcal{B}_{i}\right)+\prod_{i=1}^{M} d_{\chi_{2}, \ldots, \chi_{N}}^{\mathbb{G}, i}\left(\mathcal{A}_{i}+\mathcal{C}_{i}\right)+\prod_{i=1}^{M} d_{\chi_{2}, \ldots, \chi_{N}}^{\mathbb{G}, i}\left(\mathcal{B}_{i}+\mathcal{C}_{i}\right) .
\end{gathered}
$$

Proof

Congruence preserves Löwner partial order, so we use (3.1) and (4.2) to derive this theorem.

The following conjecture is related to Theorem 4.4, as a generalization from Conjecture 4.1. Huang et al. [14] proved that the following conjecture is true for the case of generalized matrix functions on the set of positive (semi-) definite matrices.

Conjecture 4.2

Let the hypothesis be the same as in Theorem 4.4. Then for any $r \in\{1\} \cup[2, \infty)$, we have

$$
\begin{gathered}
\prod_{i=1}^{M}\left(d_{\chi_{2}, \ldots, \chi_{N}}^{\mathbb{G}, i}\left(\mathcal{A}_{i}+\mathcal{B}_{i}+\mathcal{C}_{i}\right)\right)^{r}+\prod_{i=1}^{M}\left(d_{\chi_{2}, \ldots, \chi_{N}}^{\mathbb{G}, i}\left(\mathcal{A}_{i}\right)\right)^{r}+\prod_{i=1}^{M}\left(d_{\chi_{2}, \ldots, \chi_{N}}^{\mathbb{G}, i}\left(\mathcal{B}_{i}\right)\right)^{r}+\prod_{i=1}^{M}\left(d_{\chi_{2}, \ldots, \chi_{N}}^{\mathbb{G}, i}\left(\mathcal{C}_{i}\right)\right)^{r} \\
\geq \prod_{i=1}^{M}\left(d_{\chi_{2}, \ldots, \chi_{N}}^{\mathbb{G}, i}\left(\mathcal{A}_{i}+\mathcal{B}_{i}\right)\right)^{r}+\prod_{i=1}^{M}\left(d_{\chi_{2}, \ldots, \chi_{N}}^{\mathbb{G}, i}\left(\mathcal{A}_{i}+\mathcal{C}_{i}\right)\right)^{r}+\prod_{i=1}^{M}\left(d_{\chi_{2}, \ldots, \chi_{N}}^{\mathbb{G}, i}\left(\mathcal{B}_{i}+\mathcal{C}_{i}\right)\right)^{r} .
\end{gathered}
$$




\section{Hlawka type inequalities: generalization}

It turns out that the above results can be obtained as corollaries of a more general inequality involving $M$ positive definite tensors. For positive integers $M, K$ and $P$ with $K \leq M$, let $\mathbb{M}=\{1,2, \ldots, M\}$ and define the following symmetric sums:

$$
S_{K, M}^{P}:=\sum_{\mathbb{I} \subset \mathbb{M},|\mathbb{I}|=K}\left(\sum_{i \in \mathbb{I}} \mathcal{A}_{i}\right)^{P}
$$

The main result is the following theorem.

\section{Theorem 5.1}

Let $M \geq 3$ and $\mathcal{A}_{m} \in D_{N, I}(m=1,2, \ldots, M)$ be positive definite with an even $N$. Then for $P \in \mathbb{N}$, the inequality

$$
S_{M, M}^{P}+S_{M-2, M}^{P}+\cdots+S_{2, M}^{P} \geq S_{M-1, M}^{P}+S_{M-3, M}^{P}+\cdots+S_{1, M}^{P}
$$

holds with an even $M$, or the inequality

$$
S_{M, M}^{P}+S_{M-2, M}^{P}+\cdots+S_{1, M}^{P} \geq S_{M-1, M}^{P}+S_{M-3, M}^{P}+\cdots+S_{2, M}^{P}
$$

holds with an odd $M$.

Proof

We prove the claim by induction on $M$. The case that $M=3$ is considered in Theorem 4.1. Fix $M>4$ and suppose (5.2) or (5.3) holds for all $P$. We first assume that $M$ is even.

We now perform an induction on $P$. For $P=1$, the claim clearly holds as both sides of (5.2) or (5.3) are equal. Assume that the claim holds up to some integer $P$. Thus,

$$
S_{M, M}^{P}+S_{M-2, M}^{P}+\cdots+S_{2, M}^{P} \geq S_{M-1, M}^{P}+S_{M-3, M}^{P}+\cdots+S_{1, M}^{P} .
$$

Multiplying (i.e., taking tensor products) both sides by $\left(\mathcal{A}_{1}+\cdots+\mathcal{A}_{M}\right)$ on the right and using Proposition 3.1 (ii), we obtain

$$
\sum_{j=2: 2: M} S_{j, M}^{P+1}+\mathcal{L} \geq \sum_{j=1: 2: M-1} S_{j, M}^{P+1}+\mathcal{R}
$$

where $\mathcal{L}$ and $\mathcal{R}$ denote the respective mixed terms. Inequality (5.4) will hold if we show that $\mathcal{R} \geq \mathcal{L}$. In the following, we prove $\mathcal{R} \geq \mathcal{L}$.

For $\mathcal{L}$ and $\mathcal{R}$, some tedious multiplications yield that

$$
\begin{aligned}
& \mathcal{L}=\sum_{\substack{\mathbb{I} \subset \mathbb{M} \\
|\mathbb{|}|=M-2}}\left(\sum_{i \in \mathbb{I}} \mathcal{A}_{i}\right)^{P}\left(\sum_{i \notin \mathbb{I}} \mathcal{A}_{i}\right)+\cdots+\sum_{\substack{\mathbb{I} \subset \mathbb{M} \\
|\mathbb{I}|=2}}\left(\sum_{i \in \mathbb{I}} \mathcal{A}_{i}\right)^{P}\left(\sum_{i \notin \mathbb{I}} \mathcal{A}_{i}\right), \\
& \mathcal{R}=\sum_{\substack{\mathbb{I} \subset \mathbb{M} \\
|\mathbb{I}|=M-1}}\left(\sum_{i \in \mathbb{I}} \mathcal{A}_{i}\right)^{P}\left(\sum_{i \notin \mathbb{I}} \mathcal{A}_{i}\right)+\cdots+\sum_{k=1}^{M} \mathcal{A}_{k}^{P}\left(\sum_{i \neq k} \mathcal{A}_{i}\right) .
\end{aligned}
$$

Note that the main sums in $\mathcal{L}$ and $\mathcal{R}$ are only over even and odd sized subsets, respectively. 
The key to the proof is the following regrouping of (5.5), which reveals the underlying inductive structure:

$$
\begin{aligned}
\mathcal{R} & =\left(\sum_{i \in \mathbb{M}-\{M\}} \mathcal{A}_{i}\right)^{P} \mathcal{A}_{M}+\left(\sum_{\substack{\mathbb{I} \subset \mathbb{M}-\{M\} \\
|\mathbb{I}|=M-3}}\left(\sum_{i \in \mathbb{I}} \mathcal{A}_{i}\right)^{P}\right) \mathcal{A}_{M}+\cdots+\left(\sum_{\substack{\mathbb{I} \subset \mathbb{M}-\{M\} \\
\mathbb{I}=\{i\}}} \mathcal{A}_{i}^{P}\right) \mathcal{A}_{M} \\
& +\left(\sum_{i \in \mathbb{M}-\{M-1\}} \mathcal{A}_{i}\right)^{P} \mathcal{A}_{M-1}+\left(\sum_{\substack{\mathbb{I} \mathbb{M}-\{M-1\} \\
\mathbb{I} \mid=M-3}}\left(\sum_{i \in \mathbb{I}} \mathcal{A}_{i}\right)^{P}\right) \mathcal{A}_{M-1}+\ldots \\
& +\left(\sum_{\substack{\mathbb{I} \subset \mathbb{M}-\{M-1\} \\
\mathbb{I}=\{i\}}} \mathcal{A}_{i}^{P}\right)^{P} \mathcal{A}_{M-1} \\
& +\cdots+\cdots+\cdots+\left(\sum_{\substack{\mathbb{I} \subset \mathbb{M}-\{1\} \\
\mathbb{I}=\{i\}}} \mathcal{A}_{i}^{P}\right) \mathcal{A}_{1}
\end{aligned}
$$

and

$$
\begin{aligned}
\mathcal{L} & =\left(\sum_{\substack{i \in \mathbb{M}-\{M\} \\
|\mathbb{I}|=M-2}} \mathcal{A}_{i}\right)^{P} \mathcal{A}_{M}+\left(\sum_{\substack{\mathbb{I} \subset \mathbb{M}-\{M\} \\
\mathbb{I} \mid=M-4}}\left(\sum_{i \in \mathbb{I}} \mathcal{A}_{i}\right)^{P}\right) \mathcal{A}_{M}+\cdots+\left(\sum_{\substack{\mathbb{I} \subset \mathbb{M}-\{M\} \\
|\mathbb{I}|=2}} \mathcal{A}_{i}^{P}\right) \mathcal{A}_{M} \\
& +\cdots+\cdots+\cdots+\ldots \\
& +\left(\sum_{\substack{i \in \mathbb{M}-\{1\} \\
|\mathbb{I}|=M-2}} \mathcal{A}_{i}\right)^{P} \mathcal{A}_{1}+\left(\sum_{\substack{\mathbb{I} \subset \mathbb{M}-\{1\} \\
\mathbb{I} \mid=M-4}}\left(\sum_{i \in \mathbb{I}} \mathcal{A}_{i}\right)^{P}\right) \mathcal{A}_{1}+\cdots+\left(\sum_{\substack{\mathbb{I} \subset \mathbb{M}-\{1\} \\
|\mathbb{I}|=2}} \mathcal{A}_{i}^{P}\right) \mathcal{A}_{1} .
\end{aligned}
$$

Moreover, we have

$$
\begin{aligned}
\mathcal{R}= & \left(\sum_{j=1: 2: M-1} S_{j, \mathbb{M}-\{M\}}^{P}\right) \mathcal{A}_{M}+\left(\sum_{j=1: 2: M-1} S_{j, \mathbb{M}-\{M-1\}}^{P}\right) \mathcal{A}_{M-1} \\
& +\cdots+\left(\sum_{j=1: 2: M-1} S_{j, \mathbb{M}-\{1\}}^{P}\right) \mathcal{A}_{1}, \\
\mathcal{L}= & \left(\sum_{j=2: 2: M-2} S_{j, \mathbb{M}-\{M\}}^{P}\right) \mathcal{A}_{M}+\left(\sum_{j=2: 2: M-2} S_{j, \mathbb{M}-\{M-1\}}^{P}\right) \mathcal{A}_{M-1} \\
& +\cdots+\left(\sum_{j=2: 2: M-2} S_{j, \mathbb{M}-\{1\}}^{P}\right) \mathcal{A}_{1} .
\end{aligned}
$$

According to the hypothesis, we conclude that $\mathcal{R} \geq \mathcal{L}$.

If $M$ is odd, the only difference is in the indices of the summations, which now run over $j=1: 2: M-2$ for $\mathcal{L}$ and $j=2: 2: M-1$ for $\mathcal{R}$. We can also conclude that $\mathcal{R} \geq \mathcal{L}$. 
Theorem 5.2

Let $M \geq 3$ and $\mathcal{A}_{m} \in D_{N, I}(m=1,2, \ldots, M)$ be positive definite with an even $N$. For each $m=1,2, \ldots, M$, let $s_{m}$ be defined as

$$
s_{m}:=\sum_{1 \leq i_{1}<i_{2}<\cdots<i_{m} \leq M} d_{\chi_{2}, \ldots, \chi_{N}}^{\mathbb{G}}\left(\mathcal{A}_{i_{1}}+\mathcal{A}_{i_{2}}+\cdots+\mathcal{A}_{i_{m}}\right),
$$

where $d_{\chi_{2}, \ldots, \chi_{N}}^{\mathbb{G}}$ is the generalized tensor function defined on the set $T_{N, I}$. Then

$$
\sum_{m=1}^{M}(-1)^{M-m} s_{m, M}^{P} \geq 0
$$

\section{Remark 5.1}

Theorem 5.2 is true even when the combinatorial determinant or the permanent is replaced by the $\mathbb{G}$-immanants.

In order to obtain a plausible generalization of (5.2) or (5.3), we introduce the following denotation:

$$
\mathcal{A}_{\mathbb{J}}=\mathcal{A}_{j_{1}}+\mathcal{A}_{j_{2}}+\cdots+\mathcal{A}_{j_{\mid \mathbb{J}}},
$$

where $\mathbb{J}=\left\{j_{1}, j_{2}, \ldots, j_{|\mathbb{J}|}\right\}$ is a subset of $\mathbb{M}$ with $1 \leq j_{1}<j_{2}<\cdots<j_{|\mathbb{I}|} \leq M$.

Conjecture 5.1

Let $\mathcal{A}_{m} \in D_{N, I}(m=1,2, \ldots, M)$ be positive definite with an even $N$ and $M \geq 3$ be positive integer. Suppose that $d_{\chi_{2}, \ldots, \chi_{N}}^{\mathbb{G}}$ is the generalized tensor function defined on the set $T_{N, I}$. Then for any $r \in\{1, \ldots, M-2\} \cup$ $[M-1, \infty)$, we have

$$
\sum_{k=1}^{M}(-1)^{M-k}\left(\sum_{\mathbb{J} \subset \mathbb{M},|\mathbb{I}|=k} d_{\chi_{2}, \ldots, \chi_{N}}^{\mathbb{G}}\left(\mathcal{A}_{\mathbb{J}}\right)^{r}\right) \geq 0
$$

Remark 5.2

When $N=2$, this conjecture is proved by Huang et al. [14, Theorem 3.3].

\section{Popoviciu types inequalities}

For a convex function $f: \mathbb{R} \rightarrow \mathbb{R}$ and scalars $x_{1}, x_{2}, \ldots, x_{K}$, Jensen's inequality says that

$$
f\left(x_{1}\right)+\cdots+f\left(x_{K}\right) \geq K f\left(\frac{x_{1}+\cdots+x_{K}}{K}\right) .
$$

After Jensen's inequality, Popoviciu's inequality may be considered as the next-to-simplest inequality for convex functions, which is stated in the following lemma.

\section{Lemma 6.1}

([3, Proposition 4.1]) If $f$ is a convex function on a real interval $\mathbb{I} \subset \mathbb{R}$ and $x_{1}, x_{2}, x_{3} \in \mathbb{I}$, then

$$
f\left(x_{1}\right)+f\left(x_{2}\right)+f\left(x_{3}\right)+3 f\left(\frac{x_{1}+x_{2}+x_{3}}{3}\right) \geq 2\left(f\left(\frac{x_{1}+x_{2}}{2}\right)+f\left(\frac{x_{1}+x_{3}}{2}\right)+f\left(\frac{x_{2}+x_{3}}{2}\right)\right) .
$$

We begin with the following generalization of Lemma 6.1.

\section{Lemma 6.2}

([3, Proposition 4.2]) If $f$ is a convex function on a real interval $\mathbb{I} \subset \mathbb{R}$ and $x_{1}, x_{2}, \ldots, x_{K} \in \mathbb{I}$, then

$$
f\left(x_{1}\right)+f\left(x_{2}\right)+\cdots+f\left(x_{K}\right)+\frac{K}{K-2} f\left(\frac{x_{1}+x_{2}+\cdots+x_{K}}{K}\right) \geq \frac{2}{K-2} \sum_{1 \leq i<j \leq K} f\left(\frac{x_{i}+x_{j}}{2}\right) .
$$


Another result of this paper is given in the following theorem.

Theorem 6.1

Let $\mathcal{A}_{m} \in D_{N, I}(m=1,2, \ldots, M)$ be positive definite with an even $N$ and $M \geq 3$ be positive integer. Suppose that $d_{\chi_{2}, \ldots, \chi_{N}}^{\mathbb{G}}$ is the generalized tensor function defined on the set $T_{N, I}$. Then we have

$$
(M-2) \sum_{m=1}^{M} d_{\chi_{2}, \ldots, \chi_{N}}^{\mathbb{G}}\left(\mathcal{A}_{m}\right)+d_{\chi_{2}, \ldots, \chi_{N}}^{\mathbb{G}}\left(\sum_{m=1}^{M} \mathcal{A}_{m}\right) \geq \sum_{i<j} d_{\chi_{2}, \ldots, \chi_{N}}^{\mathbb{G}}\left(\mathcal{A}_{i}+\mathcal{A}_{j}\right) .
$$

Combining (3.2) and the following theorem, we can easily prove Theorem 6.1.

\section{Theorem 6.2}

Let $M \geq 3$ be positive integer. Suppose that $\mathcal{A}_{m} \in D_{N, I}(m=1,2, \ldots, M)$ are positive definite with an even $N$. Then for a given positive integer $P \geq 1$, we have

$$
(M-2) \sum_{m=1}^{M} \mathcal{A}_{m}^{P}+\left(\sum_{m=1}^{M} \mathcal{A}_{m}\right)^{P} \geq \sum_{i<j}\left(\mathcal{A}_{i}+\mathcal{A}_{j}\right)^{P} .
$$

Proof

The proof is similar to the one of Theorem 4.1. We proceed by introduction on $P$. For $P=1$, both sides of (6.1) are equal to $(M-1) \sum_{i=1}^{M} \mathcal{A}_{i}$; for $P=2$, we again have (6.1), since both sides of (6.1) are equal to

$$
(M-1) \sum_{i=1}^{M} \mathcal{A}_{i}^{2}+\sum_{i<j}\left(\mathcal{A}_{i} \mathcal{A}_{j}+\mathcal{A}_{j} \mathcal{A}_{i}\right)
$$

Assume that (6.1) holds for some integer $P \geq 2$. The for $P+1$, we have

$$
\begin{aligned}
& (M-2) \sum_{m=1}^{M} \mathcal{A}_{m}^{P+1}+\left(\sum_{m=1}^{M} \mathcal{A}_{m}\right)^{P+1} \\
& =\left(\sum_{m=1}^{M} \mathcal{A}_{m}\right)^{P} \sum_{m=1}^{M} \mathcal{A}_{m}+(M-2) \sum_{m=1}^{M} \mathcal{A}_{m}^{P} \sum_{m=1}^{M} \mathcal{A}_{m}^{P+1}-(M-2) \sum_{i<j}\left(\mathcal{A}_{i}^{P} \mathcal{A}_{j}+\mathcal{A}_{j}^{P} \mathcal{A}_{i}\right) \\
& \geq \sum_{i<j}\left(\mathcal{A}_{i}+\mathcal{A}_{j}\right)^{P} \sum_{k=1}^{M} \mathcal{A}_{k}-(M-2) \sum_{i<j}\left(\mathcal{A}_{i}^{P} \mathcal{A}_{j}+\mathcal{A}_{j}^{P} \mathcal{A}_{i}\right) \\
& =\sum_{i<j}\left(\mathcal{A}_{i}+\mathcal{A}_{j}\right)^{P+1}+\sum_{i<j}\left(\mathcal{A}_{i}+\mathcal{A}_{j}\right)^{P} \sum_{k \notin\{i, j\}} \mathcal{A}_{k}-(M-2) \sum_{i<j}\left(\mathcal{A}_{i}^{P} \mathcal{A}_{j}+\mathcal{A}_{j}^{P} \mathcal{A}_{i}\right) \\
& \geq \sum_{i<j}\left(\mathcal{A}_{i}+\mathcal{A}_{j}\right)^{P+1}+\sum_{i<j}\left(\mathcal{A}_{i}^{P}+\mathcal{A}_{j}^{P}\right) \sum_{k \notin\{i, j\}} \mathcal{A}_{k}-(M-2) \sum_{i<j}\left(\mathcal{A}_{i}^{P} \mathcal{A}_{j}+\mathcal{A}_{j}^{P} \mathcal{A}_{i}\right) \\
& =\sum_{i<j}\left(\mathcal{A}_{i}+\mathcal{A}_{j}\right)^{P+1} .
\end{aligned}
$$

The first inequality follows from the introduction hypothesis applied to the first two terms, while the second inequality follows from (iv) of Proposition 3.1. Now we verify the final equality as follows: Fix $i=1$, then the second term yields for each $j=2,3, \ldots, M$, product $\mathcal{A}_{1}^{P}$ with $(M-2)$ of the $\mathcal{A}_{k}$ 's $(k \neq 1)$, so for each $k \neq 1$, the product $\mathcal{A}_{1}^{P} \mathcal{A}_{k}$ occurs $(M-2)$ times, and so it also in the negative term. By symmetry, the same holds for all $i$. 
It shall be mentioned that Theorem 4.1 can be regarded as a special case of Theorem 6.2. Theorem 6.1 combined with the superadditivity inequality Proposition 3.1 for the appropriate pairs of indices implies the following corollary.

Corollary 6.1

Let $\mathcal{A}_{m} \in D_{N, I}(m=1,2, \ldots, M)$ be positive definite with an even $N$ and $M \geq 3$ be positive integer. Suppose that $d_{\chi_{2}, \ldots, \chi_{N}}^{\mathbb{G}}$ is the generalized tensor function defined on the set $T_{N, I}$. Then we have

$$
d_{\chi_{2}, \ldots, \chi_{N}}^{\mathbb{G}}\left(\sum_{m=1}^{M} \mathcal{A}_{m}\right) \geq \sum_{i \neq j}\left(d_{\chi_{2}, \ldots, \chi_{N}}^{\mathbb{G}}\left(\mathcal{A}_{i}+\mathcal{A}_{j}\right)-(M-2) d_{\chi_{2}, \ldots, \chi_{N}}^{\mathbb{G}}\left(\mathcal{A}_{i}\right)\right) .
$$

Popoviciu-Cirtoaje-Zhao inequality is an intermediate generalization of Popoviciu's inequality, which states the following.

\section{Proposition 6.1}

If $f$ is a convex function on a real interval $\mathbb{I} \subset \mathbb{R}$ and $x_{1}, x_{2}, \ldots, x_{M} \in \mathbb{I}$, then for $2 \leq K<M$,

$$
\begin{aligned}
& \left(\begin{array}{l}
M-2 \\
K-1
\end{array}\right)\left(f\left(x_{1}\right)+\cdots+f\left(x_{M}\right)\right)+M\left(\begin{array}{l}
M-2 \\
K-2
\end{array}\right) f\left(\frac{x_{1}+\cdots+x_{M}}{M}\right) \\
& \geq K \sum_{i_{1}<\cdots<i_{K}} f\left(\frac{x_{i_{1}}+x_{i_{2}}+\cdots+x_{i_{k}}}{K}\right) .
\end{aligned}
$$

From Proposition 6.1, the corresponding generalization of Theorem 6.2 is given in the following.

Theorem 6.3

Let $M \geq 3$ be positive integer. Suppose that $\mathcal{A}_{m} \in D_{N, I}(m=1,2, \ldots, M)$ are positive definite with an even $N$. Then for a given integer $P \geq 1$,

$$
\left(\begin{array}{l}
M-2 \\
K-1
\end{array}\right) \sum_{i=1}^{M} \mathcal{A}_{i}^{P}+\left(\begin{array}{c}
M-2 \\
K-2
\end{array}\right)\left(\sum_{i=1}^{M} \mathcal{A}_{i}\right)^{P} \geq \sum_{i_{1}<\cdots<i_{K}}\left(\mathcal{A}_{i_{1}}+\cdots+\mathcal{A}_{i_{K}}\right)^{P} .
$$

Combing (3.2) and the above theorem, we have the following theorem.

\section{Theorem 6.4}

Let $\mathcal{A}_{m} \in D_{N, I}(m=1,2, \ldots, M)$ be positive definite with an even $N$ and $M \geq 3$ be positive integer. Suppose that $d_{\chi_{2}, \ldots, \chi_{N}}^{\mathbb{G}}$ is the generalized tensor function defined on the set $T_{N, I}$. Then we have

$$
\begin{aligned}
& \left(\begin{array}{l}
M-2 \\
K-1
\end{array}\right) \sum_{m=1}^{M} d_{\chi_{2}, \ldots, \chi_{N}}^{\mathbb{G}}\left(\mathcal{A}_{m}\right)+\left(\begin{array}{c}
M-2 \\
K-2
\end{array}\right) d_{\chi_{2}, \ldots, \chi_{N}}^{\mathbb{G}}\left(\sum_{m=1}^{M} \mathcal{A}_{m}\right) \\
& \geq \sum_{i_{1}<\cdots<i_{K}} d_{\chi_{2}, \ldots, \chi_{N}}^{\mathbb{G}}\left(\mathcal{A}_{i_{1}}+\cdots+\mathcal{A}_{i_{K}}\right) .
\end{aligned}
$$

Instead of proving Theorem 6.3, we state the most general Popoviciu type inequality for tensors. When $N=2$, this type of the most general Popoviciu type inequality for tensors are considered in [3].

\section{Theorem 6.5}

Let $M \geq 3$ be positive integer. Suppose that $\mathcal{A}_{m} \in D_{N, I}(m=1,2, \ldots, M)$ are positive definite with an even $N$ and $S_{K, M}^{P}$ is defined as in (5.1). Then for integers $1 \leq K<L<T \leq M$,

$$
\frac{T-L}{K\left(\begin{array}{c}
M \\
K
\end{array}\right)} S_{K, M}^{P}+\frac{L-K}{T\left(\begin{array}{c}
M \\
T
\end{array}\right)} S_{T, M}^{P} \geq \frac{T-K}{L\left(\begin{array}{c}
M \\
L
\end{array}\right)} S_{L, M}^{P}
$$


The proof is omitted; it can be obtained by following the inductive technique developed above. It should be mentioned that the corresponding inequality for convex functions only holds for certain choices of $K, L$ and $T$.

\section{Theorem 6.6}

Let $\mathcal{A}_{m} \in D_{N, I}(m=1,2, \ldots, M)$ be positive definite with an even $N$ and $M \geq 3$ be positive integer. Suppose that $d_{\chi_{2}, \ldots, \chi_{N}}^{\mathbb{G}}$ is the generalized tensor function defined on the set $T_{N, I}$. Then for integers $1 \leq K<L<T \leq M$, we have

$$
\begin{aligned}
& \frac{T-L}{K\left(\begin{array}{c}
M \\
K
\end{array}\right)} \sum_{i_{1}<\cdots<i_{K}} d_{\chi_{2}, \ldots, \chi_{N}}^{\mathbb{G}}\left(\mathcal{A}_{i_{1}}+\cdots+\mathcal{A}_{i_{K}}\right)+\frac{L-K}{T\left(\begin{array}{c}
M \\
T
\end{array}\right)} \sum_{j_{1}<\cdots<j_{T}} d_{\chi_{2}, \ldots, \chi_{N}}^{\mathbb{G}}\left(\mathcal{A}_{j_{1}}+\cdots+\mathcal{A}_{j_{T}}\right) \\
& \geq \frac{T-K}{L\left(\begin{array}{c}
M \\
L
\end{array}\right)} \sum_{k_{1}<\cdots<k_{L}} d_{\chi_{2}, \ldots, \chi_{N}}^{\mathbb{G}}\left(\mathcal{A}_{k_{1}}+\cdots+\mathcal{A}_{k_{L}}\right) .
\end{aligned}
$$

\section{Acknowledgement}

The first author is supported by the Fundamental Research Funds for the Central Universities (JBK1801058). The second and third author are supported by the bilateral project between China and Serbia, "The theory of tensors, operator matrices and applications (no. 4-5)". Partial work was finished during the second author's visiting to Shanghai Key Laboratory of Contemporary Applied Mathematics in 2018.

\section{REFERENCES}

1. S. Avgustinovich, Multidimensional permanents in enumeration problems, Journal of Applied and Industrial Mathematics, vol. 4, no. 1, pp. 19-20, 2010.

2. A. Barvinok, Computing the permanent of (some) complex matrices, Foundations of Computational Mathematics, vol. 16, no. 2, pp. 329-342, 2016.

3. W. Berndt and S. Sra, Hlawka-popoviciu inequalities on positive definite tensors, Linear Algebra and its Applications, vol. 486, pp. 317-327, 2015.

4. A. Cayley, On the theory of determinants, Transaction of the Cambridge Philosophical Society, vol. 8, pp. 1-16, 1843.

5. X. Chang, V. E. Paksoy, and F. Zhang, An inequality for tensor product of positive operators and its applications, Linear Algebra and its Applications, vol. 498, pp. 99-105, 2014.

6. M. Che, C. Bu, L. Qi, and Y. Wei, Nonnegative tensors revisited: Plane stochastic tensors, Linear and Multilinear Algebra, Available online, 2018. URL: https://doi .org/10.1080/03081087.2018.1453469.

7. M. Che, L. Qi, and Y. Wei, Positive-definite tensors to nonlinear complementarity problems, Journal of Optimization Theory and Applications, vol. 168, no. 2, pp. 475-487, 2016.

8. H. Chen, G. Li, and L. Qi, SOS tensor decomposition: Theory and applications, Communications in Mathematical Sciences, vol. 14, no. 8, pp. 2073-2100, 2016.

9. W. Ding, L. Qi, and Y. Wei, Inheritance properties and sum-of-squares decomposition of hankel tensors: Theory and algorithms, BIT. Numerical Mathematics, vol. 57, no. 1, pp. 169-190, 2017.

10. S. J. Dow and P. M. Gibson, Permanents of d-dimensional matrices, Linear Algebra and its Applications, vol. 90, pp. 133-145, 1987.

11. J. Fan and A. Zhou, A semidefinite algorithm for completely positive tensor decomposition, Computational Optimization and Applications, vol. 66, no. 2, pp. 267-283, 2017.

12. W. Fechner, Hlawkas functional inequality, Aequationes Mathematicae, vol. 87, no. 1-2, pp. 71-87, 2014.

13. S. Hu, G. Li, and L. Qi, A tensor analogy of Yuan's theorem of the alternative and polynomial optimization with sign structure, Journal of Optimization Theory and Applications, vol. 168, no. 2, pp. 446-474, 2016.

14. S. Huang, C. Li, Y. Poon, and Q. Wang, Inequalities on generalized matrix functions, Linear and Multilinear Algebra, vol. 65, no. 10, pp. 1947-1961, 2017.

15. T. G. Kolda and B. W. Bader, Tensor decompositions and applications, SIAM Review, vol. 51, no. 3, pp. 455-500, 2009.

16. N. Lee and A. Cichockia, Fundamental tensor operations for large-scale data analysis in tensor train formats, arXiv preprint arXiv:1405.7786v2, 2016.

17. G. Li, L. Qi, and Y. Xu, SOS-Hankel tensors: Theory and application, arXiv: Spectral Theory, 2014.

18. L.-H. Lim, Tensors and hypermatrices. Chapter 15 in Handbook of Linear Algebra, CRC Press, Boca Raton, FL, 2 ed., 2013.

19. Z. Luo and L. Qi, Completely positive tensors: properties, easily checkable subclasses, and tractable relaxations, SIAM Journal on Matrix Analysis and Applications, vol. 37, no. 4, pp. 1675-1698, 2016. 
20. M. Marcus, Finite Dimensional Multilinear Algebra. Part II, vol. 23 of Pure and Applied Mathematics, Marcel Dekker, Inc., New York, 1975.

21. M. Marcus and H. Minc, Generalized matrix functions, Transactions of the American Mathematical Society, vol. 116, pp. 316329, 1965.

22. R. Merris, Multilinear Algebra, vol. 8 of Algebra, Logic and Applications, Gordon and Breach Science Publishers, Amsterdam, 1997.

23. R. Oldenburger, Higher dimensional determinants, The American Mathematical Monthly, vol. 47, pp. 25-33, 1940.

24. V. E. Paksoy, R. Turkmen, and F. Zhang, Inequalities of generalized matrix functions via tensor products, Electronic Journal of Linear Algebra, vol. 27, no. 1, pp. 332-341, 2014.

25. L. Qi, Eigenvalues of a real supersymmetric tensor, Journal of Symbolic Computation, vol. 40, no. 6, pp. 1302-1324, 2005.

26. L. Qi, C. Xu, and Y. Xu, Nonnegative tensor factorization, completely positive tensors, and a hierarchical elimination algorithm, SIAM Journal on Matrix Analysis and Applications, vol. 35, no. 4, pp. 1227-1241, 2014.

27. L. H. Rice, Introduction to higher determinants, Journal of Mathematics and Physics, vol. 9, pp. 47-70, 1930.

28. A. A. Taranenko, Multidimensional permanents and an upper bound on the number of transversals in Latin squares, Journal of Combinatorial Designs, vol. 23, no. 7, pp. 305-320, 2015.

29. A. A. Taranenko, Permanents of multidimensional matrices: Properties and applications, Journal of Applied and Industrial Mathematics, vol. 10, no. 4, pp. 567-604, 2016.

30. C. Xu, Z. Chen, and L. Qi, On $\{0,1\}$ CP tensors and CP pseudographs, Linear Algebra and its Applications, vol. 557, pp. 287306, 2018.

31. C. Xu, Z. Luo, L. Qi, and Z. Chen, $\{0,1\}$ completely positive tensors and multi-hypergraphs, Linear Algebra and its Applications, vol. 510, pp. 110-123, 2016. 